\title{
Bisphenol A exposure and type 2 diabetes mellitus risk: a meta-analysis
}

Semi Hwang ${ }^{1}$, Jung-eun Lim', Yoonjeong Choi ${ }^{2}$ and Sun Ha Jee ${ }^{1^{*}}$ (D)

\begin{abstract}
Background: This meta-analytic study explored the relationship between the risk of type 2 diabetes mellitus (T2DM) and bisphenol A concentrations.

Methods: The Embase and Medline (PubMed) databases were searched, using relevant keywords, for studies published between 1980 and 2018. A total of 16 studies, twelve cross-sectional, two case-control and one prospective, were included in the meta-analysis. The odds ratio (OR) and its $95 \%$ confidence interval (Cl) were determined across the sixteen studies. The OR and its $95 \% \mathrm{Cl}$ of diabetes associated with bisphenol A were estimated using both fixed-effects and random-effects models.
\end{abstract}

Results: A total of 41,320 subjects were included. Fourteen of the sixteen studies included in the analysis provided measurements of urine bisphenol A levels and two study provided serum bisphenol A levels. Bisphenol A concentrations in human bio-specimens showed positive associations with T2DM risk (OR 1.28, 95\% Cl 1.14, 1.44). A sensitivity analysis indicated that urine bisphenol A concentrations were positively associated with T2DM risk (OR 1.20, 95\% Cl 1.09, 1.31).

Conclusions: This meta-analysis indicated that Bisphenol A exposure is positively associated with T2DM risk in humans.

Keywords: Bisphenol a (BPA), Endocrine disrupting chemicals (EDCs), Diabetes mellitus (DM), Type 2 diabetes mellitus (T2DM), Hemoglobin A1c (HbA1c), Fasting plasma glucose, Obesity, Meta-analysis

\section{Background}

Type 2 diabetes mellitus (T2DM) is a metabolic disease that presents with symptoms of insulin resistance and lack of insulin [1]. The global prevalence of T2DM among adults is about 415 million, but based on projections by the International Federation of Diabetes is expected to reach 642 million in 2040 [2, 3].

Bisphenol A (BPA) is a role for endocrine disrupting chemicals (EDCs) and especially used in epoxy resin and polycarbonate plastic products such as food packaging, drink containers, and dental sealants [4-7]. Once the EDCs is deposited in the body, they can interfere with the physiological effects of estrogen, androgen and thyroid hormones by functioning as a hormone agonists and antagonists. Especially, BPA or EDCs interfere with cell signal pathways related to weight and glucose homeostasis. A number of previous experimental and epidemiological

\footnotetext{
* Correspondence: jsunha@yuhs.ac

${ }^{1}$ Department of Epidemiology and Health Promotion, Institute for Health

Promotion, Graduate School of Public Health, Yonsei University, 50-1

Yonsei-ro, Seodaemun-gu, Seoul 03722, Republic of Korea

Full list of author information is available at the end of the article
}

studies have found that EDCs can penetrate the body in several ways, including dietary intake, inhalation, skin contact, and other pathways. Thus, EDCs may have been associated with mainly the occurrence of hormone-like effects disorders and even cancers [7].

Competitive with 17- beta estradiol (E2), BPA is a type of endocrine disrupting chemical (EDCs) that disrupts estrogenic response by binding to estrogen receptors. BPA binds to androgen receptors and thyroid receptors. Unfortunately, humans are exposed to BPA through the daily exposure to BPA containing products such as canned food, plastic products, dental sealants, and household dust $[7,8]$.

In recent studies, research findings suggest that low levels of BPA can cause significant health problems. A number of scientists hypothesized that adverse health effects might be associated with high urinary BPA concentrations. Epidemiological studies have been carried out to evaluate the possible association between BPA exposure and the risk of T2DM, but the results were not consistent [9-24]. 
In this study, a meta-analysis focusing on the association between BPA concentrations (measured in urine or serum) and the risk of T2DM was performed. In addition, subgroup analyses were performed according to the sample type (urine or serum) and the study design.

\section{Methods}

\section{Study selection}

Figure 1 shows a PRISMA flow diagram that describes the selection process of this meta-analysis (Additional file 1: Table S1). As shown in the figure, the Embase and Medline (PubMed) databases were searched between 1980 and 2018 using Medical Subject Headings (MeSH) terms related to BPA and diabetes.

The keywords used in the Embase and Medline (PubMed) database searches were: Bisphenol A, BPA, 4, 4 isopropylidenediphenol or Bisphenol A bis (2 hydroxypropyl) ether dimethacrylate and Noninsulin dependent diabetes mellitus or Type 2 diabetes or Diabetes Mellitus, Type 2 or Diabetes Mellitus, Noninsulin-Dependent or Diabetes Mellitus, Ketosis-Resistant or Diabetes Mellitus, Fasting blood sugar or Fasting plasma glucose or Blood glucose, HbA1c or Glycosylated hemoglobin or Hemoglobin A1cor Glycated Hemoglobin A or Hemoglobin A, Glycated. A total of 420 articles were found: 246 were from Embase and 174 were from Medline (PubMed). First, 139 duplicated articles were removed., After, an initial review, 148 studies were excluded; 85 studies were not human research such as animal and invitro experiment, 43 studies had irrelevant exposures or outcomes and 20 studies were reviews or meta-analyses papers. Next, 133 studies were selected for full-text article review. From these studies, 117 studies, including 44 studies were not human research, 38 with irrelevant exposures or outcomes, 10 studies were reviews or meta-analyses, 22 were letter or book or comment papers, and 3 had not find full text from the same database were excluded. Finally, a total of 16 articles were included in this meta-analysis (Fig. 1).

\section{Data extraction}

Data extraction was completed twice by two reviewers, Hwang, S. and Lim, J.E. independently, with no disagreement in the selection of the final sixteen articles [9-24]. The reviewers selected the variables while considering authors, year of publication, country, type of study, type of sample, unit of measurement, population, comparison categories, and adjusted odds ratios (OR) with corresponding confidence intervals, and model adjustments. To be included in the meta-analysis, a published study had to be the original article published between 1980 and 2018. A total of 16 studies published between September, 2008 and January, 2018 were selected for final inclusion. We conducted quality assessment using the
Records identified through database search $(\mathrm{n}=420)$

- 246 from Embase

- 174 from Medline (PubMed)

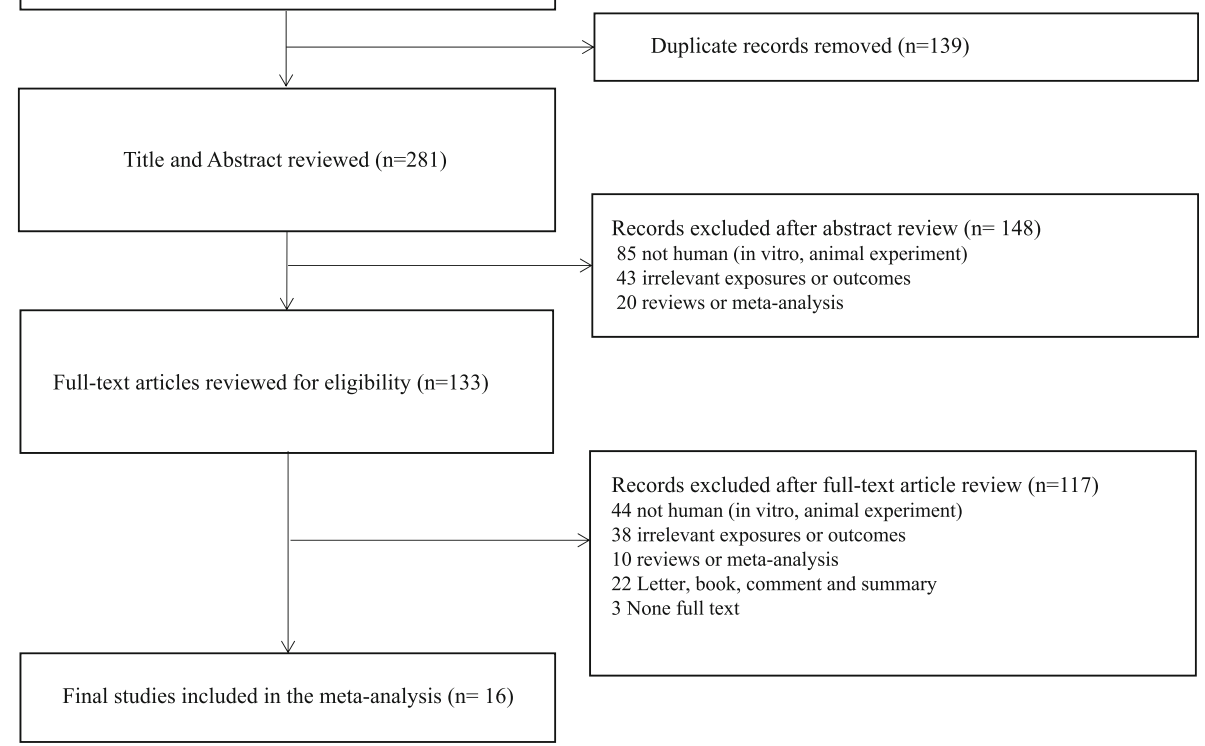

Fig. 1 A PRISMA flow diagram 
Downs and Black score [25]. The average quality score was 16 with scores ranging from 13 to 18 .

\section{Statistical analyses}

Odds ratios (OR) and 95\% confidence intervals (95\% CI) were obtained from the selected articles using the standard guidelines for meta-analysis [26]. Fixed-effects model and random-effects model were implemented. Heterogeneity was tested using the Cochrane Q-test and $\mathrm{I}^{2}$ statistic, considering an $\mathrm{I}^{2}$ value $>50 \%$ as indicative of substantial heterogeneity. A study with a significantly high OR was omitted from the meta-analysis to avoid overrepresentation. Analyses were performed by sub-groups: type of sample (serum or urine), and type of study (cross-sectional, case-control and prospective) as possible sources of heterogeneity. A Begg's Funnel Plot and an Egger's Regression Test were conducted to minimize publication bias and asymmetry of the studies. When publication bias exists, the Begg's Funnel Plot is asymmetric, or the Egger's Test $P$-value $<0.05$ [27].

To adjust for the cross-study differences between the BPA concentration units and the range of measured values, a dose-response meta-analysis (DRMA) was implemented. The dose-response meta-analyses (DRMA) was implemented by using the STATA GLST command [28] on a sample off our studies (Additional file 2: Figure S2).

Statistical analyses were performed using STATA version 13.0 software (Stata Corp, College Station, Texas).

\section{Results}

The 420 studies were searched using a systematic search strategy, referring to the PRISMA flow chart that describes the selection process of the meta-analysis [29]. After the duplicate records were removed, each article was reviewed by title, abstract, and full-text. Sixteen studies, 12 cross-sectional, 3 case-control and 1 prospective studies remained. A total of 6855 diabetic patients from among 141,320 subjects were included in the study.

Table 1 represents the characteristics of the studies included in the meta-analysis. The selected studies were performed in the USA, Korea, Iran, China and Thailand. While using funnel plot asymmetry to detect publication bias and applying Egger's regression test to measure for asymmetry, a very low publication bias was confirmed .

BPA exposure was positively associated with the risk of T2DM (Fig. 2). The pooled OR of the random-effects model was 1.28 (95\% CI, 1.14-1.44). Figure 3 presents the forest plot of sensitivity analysis after three studies were excluded, one for exhibiting highly heterogeneous results (OR 57.60; 95\% CI 21.10-157.05) [20] and two for using serum BPA concentrations [22, 24].

In Fig. 4 and Additional file 2: Figure S2, the funnel plot shows publication bias in the meta-analysis. Of the studies used in 16 final meta-analysis, only five were found to have no bias, four using urine BPA and one with serum BPA.

\section{Discussion}

In this meta-analysis, we observed that the exposure of BPA was associated with an increased risk of T2DM. Both urine and serum BPA levels were positively associated with the risk of T2DM. The results of this study showed pooled OR of 1.28 (95\% CI 1.14-1.44).

Previous studies have identified that the association between urinary BPA levels and T2DM may be biologically feasible. For example, BPA, an estrogen agonist that acts as an endocrine hormone disruptor, has been shown to be involved in several mechanisms of diabetes development including glucose homeostasis, obesity, insulin resistance, beta-cell dysfunction, inflammation, and oxidative stress [30]. BPA binding to estrogen receptors (ER) at concentrations at the physiological range or below can disrupt the pancreatic islets of Langherans, which are an essential tissue responsible for glucose metabolism [31]. BPA binding to pancreatic islet cells can induce impaired insulin or glucagon secretion, leading to an insulin-resistant state. In animal studies, adult mice exposed to low-dose BPA displayed both hyperinsulinemia and insulin resistance that are associated with pancreatic beta-cell dysfunction [32]. BPA can also act on peripheral insulin-sensitive tissues like muscle, liver, and adipose tissue [31]. Several in-vivo studies reported that BPA exposed mice showed decreased levels of circulating adiponectin as well as dysregulation of insulin signaling in skeletal muscle and liver. The mice also showed increased levels of pro-inflammatory cytokines, such as interleukin- 6 and tumor necrosis factors, which favor the development of insulin resistance [33]. Additionally, BPA has an obesogen effect resulting in the development of obesity and metabolic disorders. Sheep exposed to BPA during the prenatal period became overweight, experienced an increase in adipocyte mass, and in insulin resistance [34-36]. The induction by BPA of the insulin resistance that precedes T2DM is mainly seen when humans and animals are in a rapid growth phase. Some studies have shown that BPA exposure during pregnancy or childhood causes metabolic disorders in both humans and animals [37, 38]. However, further studies are needed to clarify the complete mechanisms of BPA exposure and T2DM risk.

Previous meta-analyses, have not implemented the dose-response analytic method used in this study to determine the relationship between BPA exposure and the risk of T2DM. A significant dose-response relationship was found between urinary BPA concentrations $(\mathrm{mg} / \mathrm{dL})$ and T2DM risk. In addition, subgroup analysis was performed according to the type of sample (urine or serum), and the type of study (cross-sectional, case-control and prospective 







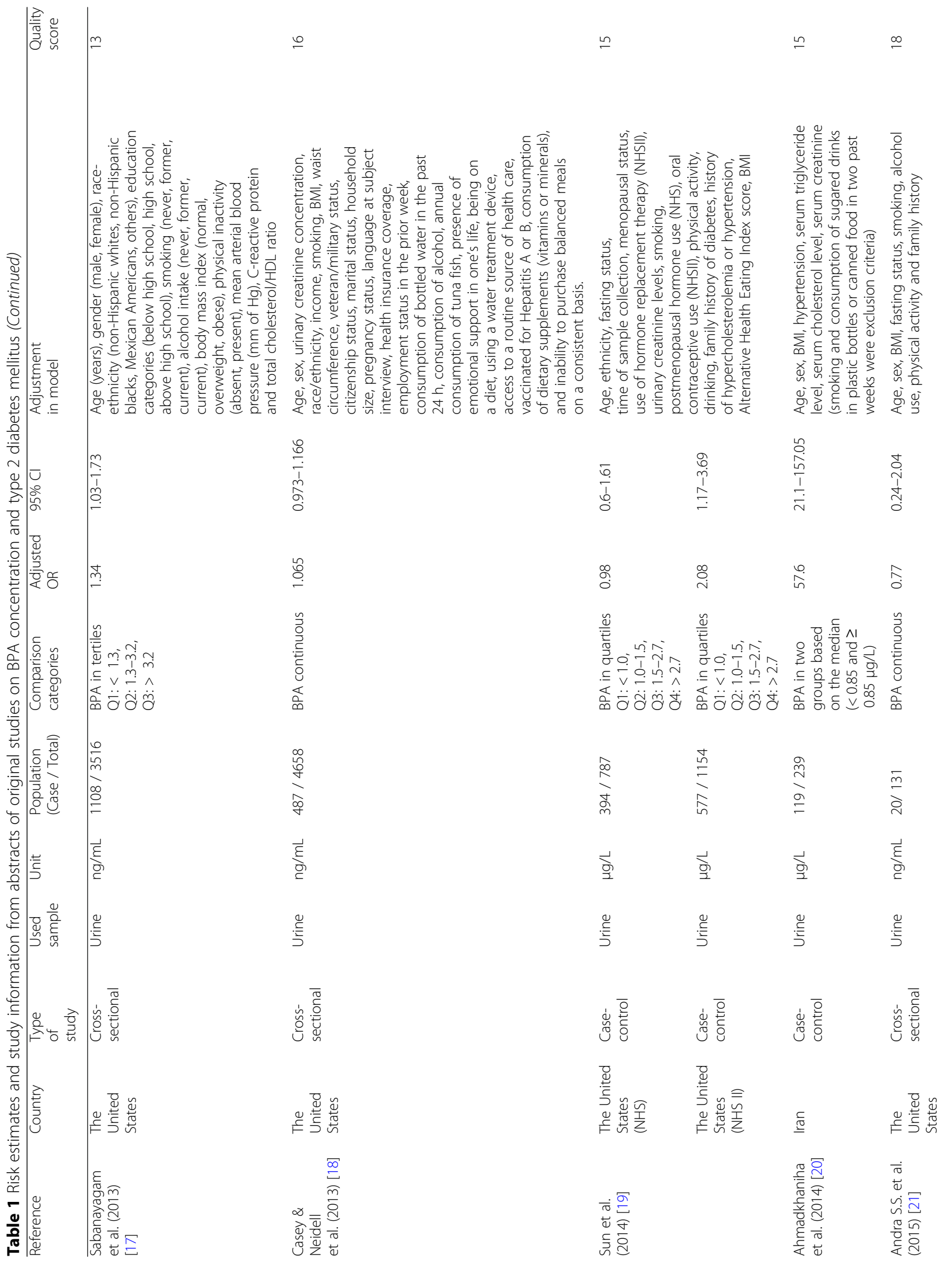




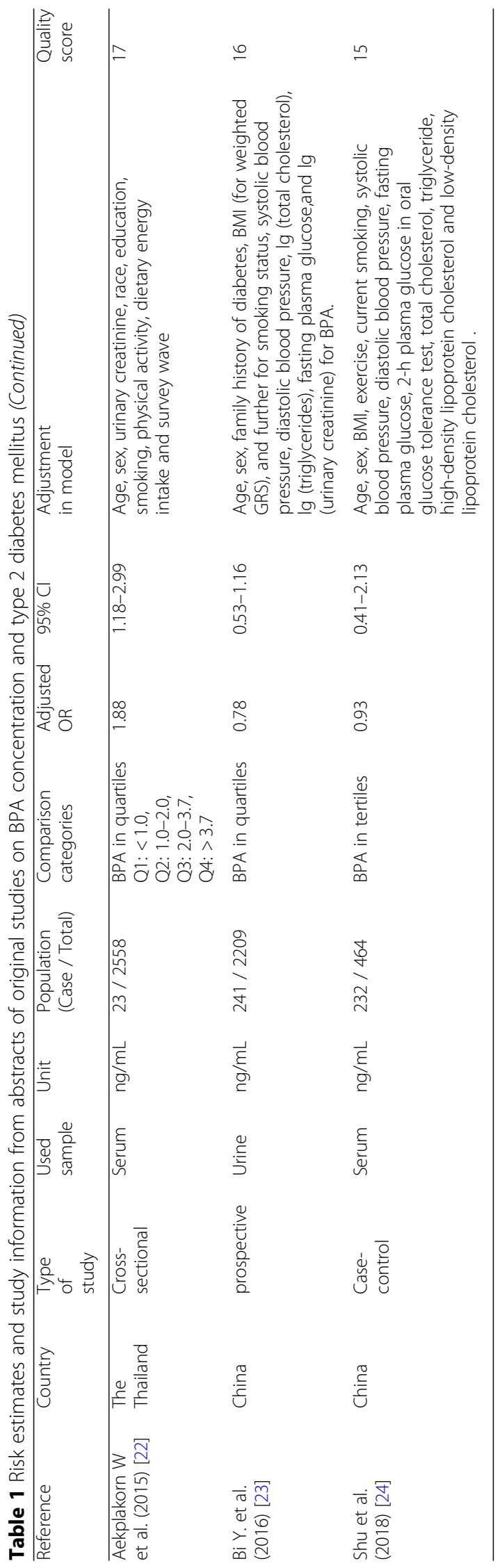




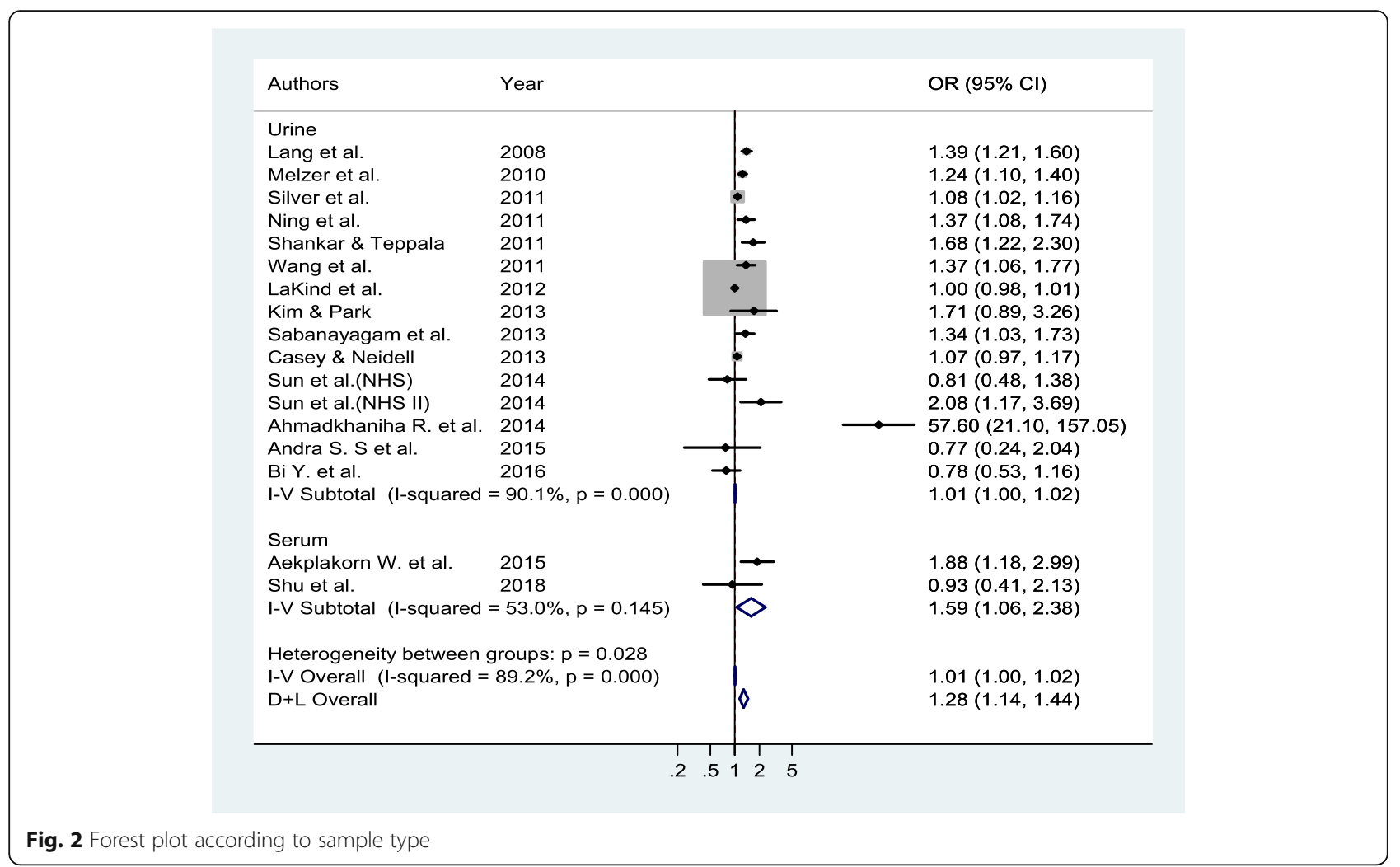

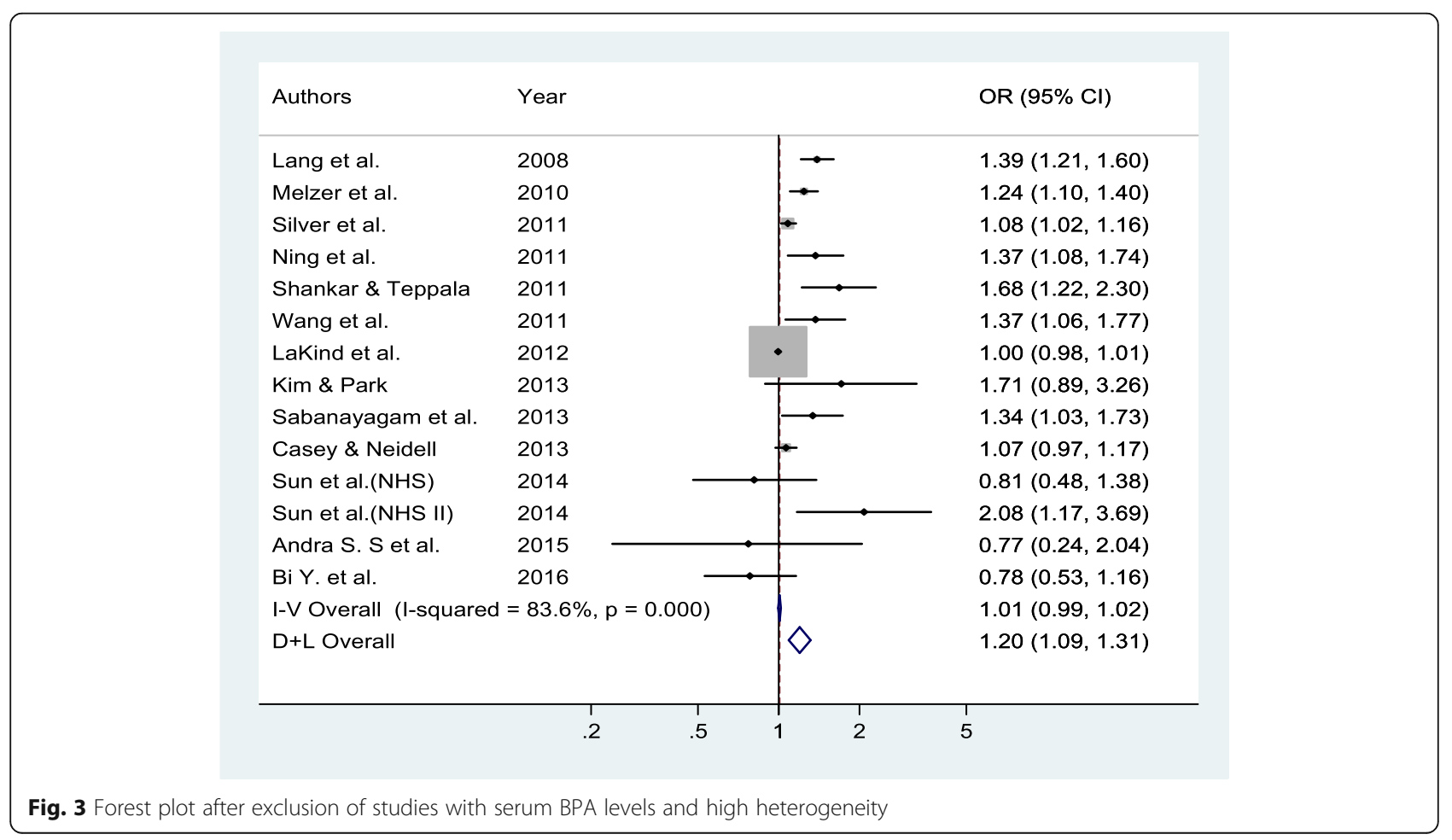


Funnel plot with pseudo 95\% confidence limits

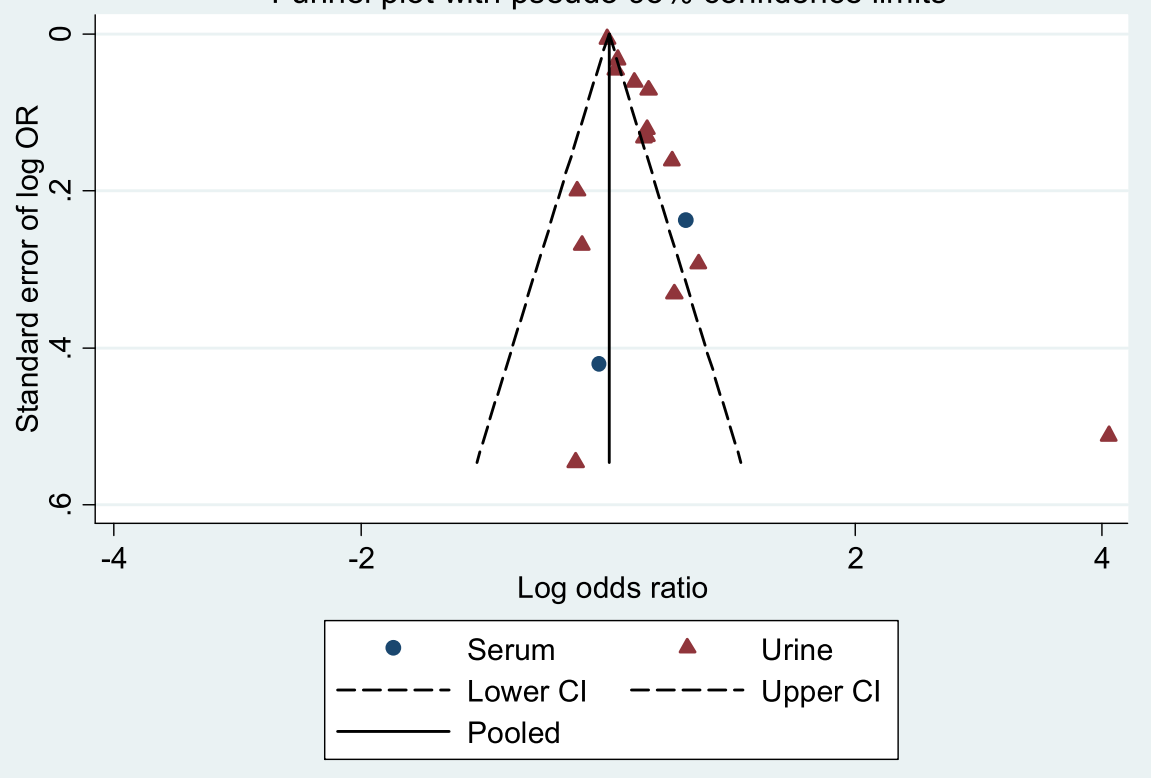

Fig. 4 Funnel plot according to sample type

study). Moreover, quality assessment methods were implemented to remove any irrelevant studies and to improve the validity of the meta-analysis. The current study considered only diabetes as a primary outcome variable as was the case with the final sixteen articles used for the meta-analysis. Despite the fact that diabetes mellitus is an important risk factor for cardiovascular disease, our study will focus specifically on T2DM, and as a result will be more focused and statistically significant than previous studies.

There were several limitations to the conduct and analysis in this study that must be considered. First, because of the limited number of cohort studies investigating the relationship between BPA exposure and T2DM risk that have been conducted, this meta-analysis included only thirteen cross-sectional, two case-control studies and one prospective studies. The inclusion of additional studies are required to validate and confirm these results. Second, this meta-analysis included fourteen studies which used spot urinary BPA concentrations and two study that used serum BPA concentrations as a surrogate marker of BPA exposure. It is unclear whether spot urinary BPA concentrations could accurately reflect the long term exposure level of BPA in individuals. Spot urinary $\mathrm{BPA}$ concentrations are the most commonly used method to assess BPA exposure levels because of it is short half-life and the convenience of the measurement method $[11,20]$. Although some studies have demonstrated that spot urine samples can reasonably predict long-term exposures in adults $[39,40]$, the validity of such results still needs to be proven. Some recent epidemiological studies used serum BPA concentrations to investigate the health effects of BPA [22, 40]. In these studies, the authors explained that serum BPA could be an appropriate surrogate for BPA exposure because serum BPA reflected the true levels of active BPA [22]. There is not sufficient information to determine the most suitable method for measuring BPA concentrations (e.g. spot urinary BPA concentrations, 24-h urinary BPA concentrations, serum BPA concentration) that accurately reflect the level of BPA exposure. Third, although linear relationships between BPA exposure and risk of T2DM were tested in this meta-analysis, several studies have suggested inverted U-shape or non-linear relationships $[19,21,22]$. To clarify this complex dose-response relationship, more detailed research is required. Fourth, a random effects model was implemented after performing statistical heterogeneity tests because of the significant effect that heterogeneity in inclusive studies could have on the meta-analystic results.

\section{Conclusions}

In conclusion, this meta-analysis demonstrated that BPA concentrations measured in urine or serum is positively associated with T2DM risk. Furthermore, prospective cohort studies, including carefully collected data about the dietary sources of BPA exposure and potential confounding, will help clarify the role of BPA in the pathogenesis of diabetes. 


\section{Additional files}

Additional file 1: PRISMA 2009 Checklist. Preferred report items for systematic review and meta-analysis were identified through a checklist. (DOC 64 kb)

Additional file 2: Figure S1. Funnel plot with egger. Figure S2. GLST These are additional sub-analysis results. (PPTX $44 \mathrm{~kb}$ )

\section{Abbreviations}

BPA: Bisphenol A; Cl: Confidence interval; EDC: Endocrine disrupting chemicals; OR: Odds ratio; T2DM : Type 2 diabetes mellitus

\section{Acknowledgements}

Not applicable

\section{Funding}

This research was funded by a grant (15162MFDS631) from the Ministry of Food and Drug Safety in 2015.

\section{Availability of data and materials}

The datasets used and/or analysed during the current study available from the first or corresponding author on reasonable request.

\section{Authors' contributions}

HS, LJE and JSH designed the study. HS collected the data and did the meta-analysis. HS, LJE contributed equally to the manuscript. CY discussed biological mechanism of in manuscript. HS, LJE and JSH contributed to study design and critically reviewed the paper. All authors read and approved the final manuscript.

\section{Ethics approval and consent to participate}

Not applicable

\section{Consent for publication}

Not applicable

\section{Competing interests}

The authors declare that they have no competing interests.

\section{Publisher's Note}

Springer Nature remains neutral with regard to jurisdictional claims in published maps and institutional affiliations.

\section{Author details}

${ }^{1}$ Department of Epidemiology and Health Promotion, Institute for Health Promotion, Graduate School of Public Health, Yonsei University, 50-1 Yonsei-ro, Seodaemun-gu, Seoul 03722, Republic of Korea. ${ }^{2}$ Department of Public Health, Graduate School, Yonsei University, Seoul, Republic of Korea.

\section{Received: 22 June 2018 Accepted: 22 October 2018}

\section{Published online: 06 November 2018}

\section{References}

1. Chamberlain JJ, Rhinehart AS, Shaefer CF Jr, Neuman A. Diagnosis and Management of Diabetes: synopsis of the 2016 American Diabetes Association standards of medical Care in Diabetes. Ann Intern Med. 2016; 164(8):542-52.

2. American Diabetes Association. Classification and diagnosis of diabetes. In: 2016 Standards of Medical Care in Diabetes. Diabetes Care. 2016:39:S13-22.

3. Ogurtsova K, da Rocha Fernandes JD, Huang Y, Linnenkamp U, Guariguata L, Cho NH, Cavan D, Shaw JE, Makaroff LE. IDF diabetes atlas: global estimates for the prevalence of diabetes for 2015 and 2040. Diabetes Res Clin Pract. 2017;128:40-50.

4. Arenholt-Bindslev D, Breinholt V, Preiss A, Schmalz G. Time-related bisphenol-a content and estrogenic activity in saliva samples collected in relation to placement of fissure sealants. Clin Oral Investig. 1999:3(3):120-5.

5. Sajiki J, Yonekubo J. Leaching of bisphenol a (BPA) to seawater from polycarbonate plastic and its degradation by reactive oxygen species. Chemosphere. 2003;51(1):55-62.
6. Calafat AM, Kuklenyik Z, Reidy JA, Caudill SP, Ekong J, Needham LL. Urinary concentrations of bisphenol a and 4-nonylphenol in a human reference population. Environ Health Perspect. 2005;113(4):391-5.

7. Stojanoska MM, Milosevic N, Milic N, Abenavoli L. The influence of phthalates and bisphenol a on the obesity development and glucose metabolism disorders. Endocrine. 2017:55(3):666-81.

8. Hu Y, Wen S, Yuan D, Peng L, Zeng R, Yang Z, Liu Q, Xu L, Kang D. The association between the environmental endocrine disruptor bisphenol a and polycystic ovary syndrome: a systematic review and meta-analysis. Gynecol Endocrinol. 2018;34(5):370-7.

9. Lang IA, Galloway TS, Scarlett A, Henley WE, Depledge M, Wallace RB, Melzer D. Association of urinary bisphenol a concentration with medical disorders and laboratory abnormalities in adults. JAMA. 2008;300(11):1303-10.

10. Melzer D, Rice NE, Lewis C, Henley WE, Galloway TS. Association of urinary bisphenol a concentration with heart disease: evidence from NHANES 2003/ 06. PLoS One. 2010;5:e8673.

11. Silver MK, O'Neill MS, Sowers MR, Park SK. Urinary bisphenol a and type-2 diabetes in US adults: data fromNHANES 2003-2008. PLoS One. 2011;6:e26868.

12. Ning $\mathrm{G}$, et al. Relationship of urinary bisphenol a concentration to risk for prevalent type 2 diabetes in Chinese adults: a cross-sectional analysis. Ann Intern Med. 2011:155:368-74

13. Shankar A, Teppala S. Relationship between urinary bisphenol a levels and diabetes mellitus. J Clin Endocrinol Metab. 2011;96(12):3822-6.

14. Wang T, Li M, Chen B, Xu M, Xu Y, Huang Y, Lu J, Chen Y, Wang W, Li X, Liu Y, Bi Y, Lai S, Ning G. Urinary bisphenol a (BPA) concentration associates with obesity and insulin resistance. J Clin Endocrinol Metab. 2012;97(2):E223-7.

15. LaKind JS, Goodman M, Naiman DQ. Use of NHANES data to link chemical exposures to chronic diseases: a cautionary tale. PLoS One. 2012;7(12): e51086.

16. Kim K, Park H. Association between urinary concentrations of bisphenol a and type 2 diabetes in Korean adults: a population-based cross-sectional study. Int J Hyg Environ Health. 2013;216(4):467-71.

17. Sabanayagam C, Teppala S, Shankar A. Relationship between urinary bisphenol a levelsand prediabetes among subjects free of diabetes. Acta Diabetol. 2013;50:625-31.

18. Casey MF. Disconcordance in statistical models of bisphenol a and chronic disease outcomes in NHANES 2003-08. PLoS One. 2013;8(11):e79944.

19. Sun $\mathrm{Q}$, et al. Association of urinary concentrations of bisphenol a and phthalate metabolites with risk of type 2 diabetes: a prospective investigation in the nurses' health study (NHS) and NHSII cohorts. Environ Health Perspect. 2014;122:616-23.

20. Ahmadkhaniha $R$, Mansouri M, Yunesian M, Omidfar K, Jeddi MZ, Larijani B, Mesdaghinia A, Rastkari N. Association of urinary bisphenol a concentration with type-2 diabetes mellitus. J Environ Health Sci Eng. 2014;12(1):64.

21. Andra SS, Kalyvas H, Andrianou XD, Charisiadis P, Christophi CA, Makris KC. Preliminary evidence of the association between monochlorinated bisphenol a exposure and type II diabetes mellitus: a pilot study. J Environ Sci Health A Tox Hazard Subst Environ Eng. 2015;50(3):243-59.

22. Aekplakorn W, Chailurkit LO, Ongphiphadhanakul B. Association of serum bisphenol a with hypertension in thai population. Int J Hypertens. 2015:594189.

23. Bi Y, Wang W, Xu M, Wang T, Lu J, Xu Y, Dai M, Chen Y, Zhang D, Sun W, Ding L, Chen Y, Huang X, Lin L, Qi L, Lai S, Ning G. Diabetes genetic risk score modifies effect of bisphenol a exposure on deterioration in glucose metabolism. J Clin Endocrinol Metab. 2016;101(1):143-50.

24. Shu X, Tang S, Peng C, Gao R, Yang S, Luo T, Cheng Q, Wang Y, Wang Z, Zhen Q, Hu J, Li Q. Bisphenol a is not associated with a 5-year incidence of type 2 diabetes: a prospective nested case-control study. Acta Diabetol. 2018;55(4):369-75

25. Dowans and Black. The feasibility of creating a checklist for the assessment of the methodological quality both of randomised and non-randomised studies of health care interventions. J Epidemiol Community Health. 1998; 52:377-384.

26. Borenstein M, Hedges LV, Higgins JPT, Rothstein HR. Introduction to MetaAnalysis. Wiley; 2009.

27. Begg CB, Mazumdar M. Operating characteristics of a rank correlation test for publication bias. Biometrics. 1994;50(4):1088-101.

28. Shim SR, Shin IS, Yoon BH, Bae JM. Dose-response meta-analysis using STATA software. J Health Info Stat. 2016:41(3):351-8.

29. Moher D, Liberati A, Tetzlaff J, Altman DG, The PRISMA. Preferred reporting items for systematic reviews and meta-analyses: the PRISMA statement. PLoS Med. 2009;6(7):e1000097. 
30. Takeuchi T, Tsutsumi O, Ikezuki Y, et al. Positive relationship between androgen and the endocrine disruptor, bisphenol a, in normal women and women with ovarian dysfunction. Endocr J. 2004;21:165-9.

31. Alonso-Magdalena P, Morimoto S, Ripoll C, Fuentes E, Nadal A. The estrogenic effect of bisphenol a disrupts pancreatic beta-cell function in vivo and induces insulin resistance. Environ Health Perspect. 2006;114(1): 106-12.

32. Ariemma F, D'Esposito V, Liguoro D, Oriente F, Cabaro S, Liotti A, et al. Lowdose bisphenol-a impairs Adipogenesis and generates dysfunctional 3T3-L1 adipocytes. PLoS One. 2016;11(3):e0150762.

33. Moon MK, Jeong IK, Jung OT, Ahn HY, Kim HH, Park YJ, et al. Long-term ora exposure to bisphenol a induces glucose intolerance and insulin resistance. J Endocrinol. 2015;226:35-42.

34. Yadav A, Kataria MA, Saini V, Yadav A. Role of leptin and adiponectin in insulin resistance. Clin Chim Acta. 2013:417:80-4.

35. Trujillo ME, Scherer PE. Adiponectin-journey from an adipocyte secretory protein to biomarker of the metabolic syndrome. J Intern Med. 2005;257: $167-75$.

36. Veiga-Lopez A, Moeller J, Sreedharan R, Singer K, Lumeng CN, Ye W, et al. Developmental programming: interaction between prenatal BPA exposure and postnatal adiposity on metabolic variables in female sheep. Am J Physiol Endocrinol Metab. 2015;310:E238-47.

37. Menale C, Piccolo MT, Cirillo G, Calogero RA, Papparella A, Mita L, et al. Bisphenol A effects on gene expression in adipocytes from children: association with metabolic disorders. J Mol Endocrinol. 2015;54(3):289-303.

38. Chou WC, Chen JL, Lin CF, Chen YC, Shih FC, Chuang CY. Biomonitoring of bisphenol a concentrations in maternal and umbilical cord blood in regard to birth outcomes and adipokine expression:a birth cohort study in Taiwan. Environ Health A Glob Access Sci Source. 2011;10:94.

39. Mahalingaiah S, Meeker JD, Pearson KR, Calafat AM, Ye X, Petrozza J, Hauser R. Temporal variability and predictors of urinary bisphenol a concentrations in men and women. Environ Health Perspect. 2008;1 16(2):173-8.

40. Lin CY, Shen FY, Lian GW, Chien KL, Sung FC, Chen PC, Su TC. Association between levels of serum bisphenol a, a potentially harmful chemical in plastic containers, and carotid artery intima-media thickness in adolescents and young adults. Atherosclerosis. 2015;241(2):657-63.

Ready to submit your research? Choose BMC and benefit from:

- fast, convenient online submission

- thorough peer review by experienced researchers in your field

- rapid publication on acceptance

- support for research data, including large and complex data types

- gold Open Access which fosters wider collaboration and increased citations

- maximum visibility for your research: over $100 \mathrm{M}$ website views per year

At $\mathrm{BMC}$, research is always in progress.

Learn more biomedcentral.com/submissions 